\title{
A Modified NHL-BFM-95 Regimen Produces Better Outcome Than HyperCVAD in Adult Patients with T-Lymphoblastic Lymphoma, a Two-Institution Experience
}

\section{Chun Li, MS ${ }^{1}$ \\ Zhi-Jun Wuxiao, MD² \\ Xiaoqin Chen, $\mathrm{MD}^{1}$ \\ Guanjun Chen, $\mathrm{MS}^{1}$ \\ Yue Lu, PhD' \\ Zhongjun Xia, MD ${ }^{1}$ \\ Yang Liang, $\mathrm{MD}^{1}$ \\ Hua Wang, $M D^{1}$}

*A list of author's affiliations appears at the end of the paper.

Correspondence: Hua Wang, MD

Department of Hematologic Oncology,

State Key Laboratory of Oncology in South China/ Cancer Center, Collaborative Innovation Center for Cancer Medicine, Sun Yat-sen University, Guangzhou, Guangdong 510060, China

Tel: 86-20-87342462

Fax: 86-20-87342462

E-mail: wanghua@sysucc.org.cn

Co-correspondence: Yang Liang, MD Department of Hematologic Oncology, State Key Laboratory of Oncology in South China/ Cancer Center, Collaborative Innovation Center for Cancer Medicine, Sun Yat-sen University, Guangzhou, Guangdong 510060, China

Tel: 86-20-87342462

Fax: 86-20-87342462

E-mail: liangyang@sysucc.org.cn

Co-correspondence: Zhongjun Xia, MD Department of Hematologic Oncology, State Key Laboratory of Oncology in South China/ Cancer Center, Collaborative Innovation Center for Cancer Medicine, Sun Yat-sen University, Guangzhou, Guangdong 510060, China

Tel: 86-20-87342438

Fax: 86-20-87342438

E-mail: xiazhj@sysucc.org.cn

Received September 22, 2019

Accepted December 5, 2019

Published Online December 6, 2019

${ }^{*}$ Chun Li, Zhi-Jun Wuxiao, and Xiaoqin Chen contributed equally to this work.

\section{Purpose}

Lymphoblastic lymphoma (LBL) is an invasive neoplasm of precursor T-cell or B-cell lineage. A broadly accepted standard treatment for adult LBL has not yet been defined.

\section{Materials and Methods}

To address this issue, we compared two chemotherapy regimens: a modified non-Hodgkin lymphoma Berlin-Frankfurt-Münster-95 (NHL-BFM-95) regimen and HyperCVAD/MA. This retrospective study consecutively enrolled 207 adult LBL patients at two hospitals from 2000 to 2018. Univariate and multivariate analysis were used to assess prognostic factors.

\section{Results}

In the present study, most clinical characteristics were similar between the two treatment groups except for age and lactate dehydrogenase (LDH) level. Patients treated with modified NHL-BFM-95 regimen tended to be younger and with elevated LDH level. The modified NHLBFM-95 regimen produced better treatment outcomes than those with HyperCVAD/MA in patients with T-LBL or patients $<40$ years. Treatment with HyperCVAD/MA, high Eastern Cooperative Oncology Group scores, and bone marrow involvement were independent risk factors in T-LBL. No patients interrupted treatment for severe adverse events.

\section{Conclusion}

The results suggested that the modified regimen is well-tolerated and can produce the promising outcomes in patients with T-LBL or patients $<40$ years.

\author{
Key words \\ Lymphoblastic lymphoma, Adult, Prognosis, Treatment, \\ Modified NHL-BFM-95 regimen
}




\section{Introduction}

Lymphoblastic lymphoma (LBL) is an invasive neoplasm resembling acute lymphoblastic leukemia [1]. It is of precursor T-cell (T-LBL) or B-cell (B-LBL) lineage [2]. Despite the great histologic similarities between T-LBL and B-LBL, there are still some different clinical features between T-LBL and B-LBL. T-LBL is featured by mediastinal and lymph node invasion. Compared with T-LBL, B-LBL has yet to be extensively studied for B-LBL is a rare disease, accounting for $10 \%$ $20 \%$ of LBL patients. The clinical features of B-LBL have not been fully reported, especially in adult patients. Most studies on B-LBL are focused on pediatric patients [3]. Current treatment strategies of LBL are based on intensive multidrug ALL-type chemotherapy. The conventional or intensive chemotherapy protocols for non-Hodgkin lymphoma (NHL) have produced relatively poor outcomes [4,5], therefore were abandoned. Multiple studies have reported that improvements in long-term outcome were achieved with ALL-type protocols in LBL [6-8]. In 2010, the non-Hodgkin lymphoma Berlin-Frankfurt-Münster-95 (NHL-BFM-95) regimen was modified in our department and used to treat adult patients with LBL; in the meanwhile, a standard hyperfractionated cyclophosphamide, vincristine, adriamycin, dexamethasone/ methotrexate, and cytarabine (HyperCVAD/MA) was also applied to adult patients with LBL. The purpose of the present study was to further analyze the clinical characteristics and prognostic factors in LBL and to evaluate two regimens (HyperCVAD and modified NHL-BFM-95) used to treat adult LBL.

\section{Materials and Methods}

\section{Study group}

This study included a total of 207 consecutive patients newly diagnosed with LBL at two hospitals in China (Sun Yat-sen University Cancer Center and The First Affiliated Hospital of Hainan Medical College) from January 2000 to August 2018. The inclusion criteria were as follows: (1) patients pathologically diagnosed with LBL based on the revised European-American lymphoma classification [3]; (2) patients over 18 years old; (3) patients at any stage with normal internal organ functions, unless directly altered by the disease. The exclusion criteria were as follows: (1) patients with $\mathrm{Ph}-$ positive B-LBL was excluded from the study for the unique clinical characteristics and treatment strategies; (2) according to World Health Organization 2016 classification, patients who had more than $20 \%$ blasts in the bone marrow (BM) were defined as ALL and were excluded from this study; (3) patients with severe immunodeficiency or pre-existing diseases preventing chemotherapy. The clinical stage was assessed according to the Ann Arbor NHL system. Information was derived from pretreatment evaluations and re-evaluations. Eastern Cooperative Oncology Group (ECOG) score was used to evaluate the patients' performance status. Central nervous system (CNS) invasion was diagnosed by blast cells discovered in the cerebrospinal fluid or cerebral infiltration on imaging examination. BM invasion was defined as $>5 \%$ blasts in the BM. According to international prognostic index (IPI), patients were classified into low-risk group (IPI $<3$ ) and high-risk group (IPI $\geq 3$ ). Adolescent and young adult (AYA) is usually defined as individuals $15-39$ years old. In this study, patients $<40$ years was considered a distinct age group.

\section{Treatment}

The HyperCVAD/MA regimen in the present study was the standard regimen have been reported previously [6]. The regimen included eight cycles of induction-consolidation courses alternating HyperCVAD with high-dose methotrexate (HD-MTX) and cytarabine and a maintenance phase. Mediastinal irradiation was recommended for patients with mediastinal disease. Maintenance therapy lasted for 24 months. The details of induction/consolidation/maintenance phases were described in S1 Table.

The modified NHL-BFM-95 protocol was originated from NHL-BFM-95 regimen. The following differences were observed: (1) to avoid cardiotoxicity, pirarubicin replaced daunorubicin in induction protocol $1 \mathrm{~A}$ and the reinduction protocol 2A; (2) due to the modification of polyethylene gly$\mathrm{col}(\mathrm{PEG})$, pegaspargase overcomes the immunogenicity and severe allergic reaction of asparaginase, and has a longer half-live; so, we substituted pegaspargase for L-asparaginase as the inductive drug in induction protocol IA and reinduction protocol IIA; (3) a routine intrathecal chemotherapy and additional four HD-MTX treatments during maintenance phase instead of intracranial prophylactic radiotherapy to prevent CNS invasion. (4) Citrovorum folinate was administered at a dose of $15 \mathrm{mg} / \mathrm{m}^{2}$ at $36,42,48,54,60,66$, and 72 hours after high-dose methotrexate.

Schemes of induction, $\mathrm{M}$, reinduction, and maintenance treatment were described in Table 1. Treatment lasted for 30 months.

Rituximab was not used in the B-LBL cohort. 
Table 1. Protocols of modified NHL-BFM-95 regimen

\begin{tabular}{|c|c|c|}
\hline Drug & Dose & Days of administration \\
\hline \multicolumn{3}{|l|}{ Induction protocol IA } \\
\hline Prednisone (PO) & $60 \mathrm{mg} / \mathrm{m}^{2}$ per day & $1-28$, then taper over $3 \times 3$ days \\
\hline Vincristine (IV) & $1.5 \mathrm{mg} / \mathrm{m}^{2}(\max 2 \mathrm{mg})$ & $8,15,22,29$ \\
\hline Pirarubicin (IV) & $30 \mathrm{mg} / \mathrm{m}^{2}$ & $8,15,22,29$ \\
\hline Pegaspargase (IM) & $2,500 \mathrm{IU} / \mathrm{m}^{2}$ & 15,29 \\
\hline IT chemotherapy ${ }^{\mathrm{a})}$ & & $1,12,33$ \\
\hline \multicolumn{3}{|l|}{ Induction protocol IB } \\
\hline Cyclophosphamide (IV) & $1,000 \mathrm{mg} / \mathrm{m}^{2}$ per dose & 36,64 \\
\hline Cytarabine (IV) & $75 \mathrm{mg} / \mathrm{m}^{2}$ & $38-41,45-48,52-55,59-62$ \\
\hline 6-Mercaptopurine (PO) & $60 \mathrm{mg} / \mathrm{m}^{2}$ & $36-63$ \\
\hline IT chemotherapy ${ }^{\mathrm{a})}$ & & 45,59 \\
\hline \multicolumn{3}{|l|}{ Protocol M } \\
\hline 6-Mercaptopurine (oral) & $25 \mathrm{mg} / \mathrm{m}^{2}$ per day & $1-56$ \\
\hline Methotrexate & $5 \mathrm{~g} / \mathrm{m}^{2}$ & $8,22,36,50$ \\
\hline Methotrexate (IT) & & $8,22,36,50$ \\
\hline \multicolumn{3}{|l|}{ Reinduction protocol IIA } \\
\hline Dexamethasone (PO) & $60 \mathrm{mg} / \mathrm{m}^{2}$ per day & $1-21$, then taper over $3 \times 3$ days \\
\hline Vincristine (IV) & $1.5 \mathrm{mg} / \mathrm{m}^{2}(\max 2 \mathrm{mg})$ & $8,15,22,29$ \\
\hline Pirarubicin (IV) & $30 \mathrm{mg} / \mathrm{m}^{2}$ & $8,15,22,29$ \\
\hline Pegaspargase (IM) & $2,500 \mathrm{IU} / \mathrm{m}^{2}$ & 8 \\
\hline \multicolumn{3}{|l|}{ Reinduction protocol IIB } \\
\hline Cyclophosphamide (IV) & $1,000 \mathrm{mg} / \mathrm{m}^{2}$ per dose & 36 \\
\hline Cytarabine (IV) & $75 \mathrm{mg} / \mathrm{m}^{2}$ per dose & $38-41,45-48$ \\
\hline 6-Thioguanine (PO) & $60 \mathrm{mg} / \mathrm{m}^{2}$ per day & $36-49$ \\
\hline IT chemotherapy ${ }^{\mathrm{a})}$ & & 45,59 \\
\hline \multicolumn{3}{|l|}{ Maintenance therapy } \\
\hline Methotrexate (PO) & $20 \mathrm{mg} / \mathrm{m}^{2}$ per dose & Once a week \\
\hline 6-Mercaptopurine (PO) & $50 \mathrm{mg} / \mathrm{m}^{2}$ per day & Daily \\
\hline Methotrexate $^{\text {b) }}$ & $5 \mathrm{~g} / \mathrm{m}^{2}$ & 4 doses, 3-mo intervals \\
\hline
\end{tabular}

NHL-BFM-95, non-Hodgkin lymphoma Berlin-Frankfurt-Münster-95; PO, oral; IV, intravenously; IM, intramuscularly; IT, intrathecal. ${ }^{\mathrm{a}}$ Intrathecal (IT) drugs: methotrexate $\left(15 \mathrm{mg} / \mathrm{m}^{2}\right)$, cytarabine $\left(40 \mathrm{mg} / \mathrm{m}^{2}\right)$, and dexamethasone (4 mg), b) Onetenth of the methotrexate was administered within the first 0.5 hours, and the rest was given through IV drip over 23.5 hours.

\section{Response assessment}

The response was assessed after each chemotherapy session (e.g., induction I and II). After the chemotherapy was completed, the clinical response was assessed every 3 months for the first 2 years after treatment and afterward every half a year for the next 3 years. Complete remission (CR) was described as the normalization of any abnormal lymphadenopathy through physical and imaging examinations. For patients with initial BM invasion, CR was described as less than $5 \%$ of the blasts though BM aspiration. Minimal residual disease (MRD) was tested after consolidation regimen via multi-parametric flow cytometry with an eight-color panel, and the sensitivity was $0.01 \%$. Partial remission was described as a $70 \%$ to $50 \%$ reduction in tumor size. Progression was described as an increase of $>25 \%$ in residual tumor size.

\section{Statistical analysis}

Clinical features were compared using two-tailed chisquare tests, Fisher exact test, or Wilcoxon rank-sum test between two subtypes. Overall survival (OS) was estimated from diagnosis to death from any cause. Progression-free survival (PFS) was described as the time from diagnosis to the primary occurrence of progression or death from any cause. Patients who did not experience any of these events or lost to follow-up were censored. Estimates of PFS and OS distributions were calculated by the Kaplan-Meier method and compared by the log-rank test. Prognostic factors were 
Table 2. Baseline characteristics of patients in two differently treated group

\begin{tabular}{|c|c|c|c|}
\hline Parameter & Modified NHL-BFM-95 & HyperCVAD/MA & p-value \\
\hline Total & 136 & 71 & \\
\hline \multicolumn{4}{|l|}{ Age (yr) } \\
\hline$<40$ & $109(80.1)$ & $46(64.8)$ & 0.016 \\
\hline$\geq 40$ & 27 (19.9) & $25(35.2)$ & \\
\hline \multicolumn{4}{|l|}{ Sex } \\
\hline Male & $87(64.0)$ & 47 (66.2) & 0.750 \\
\hline Female & $49(36.0)$ & $24(33.8)$ & \\
\hline \multicolumn{4}{|c|}{ Immunophenotype } \\
\hline B-LBL & $29(21.3)$ & $21(29.6)$ & 0.188 \\
\hline T-LBL & $107(78.7)$ & $50(70.4)$ & \\
\hline \multicolumn{4}{|l|}{ ECOG } \\
\hline$\leq 2$ & $106(77.9)$ & $57(80.3)$ & 0.982 \\
\hline$>2$ & $30(22.1)$ & $14(19.7)$ & \\
\hline \multicolumn{4}{|l|}{ Stage } \\
\hline $\mathrm{I} / \mathrm{II}$ & $22(16.2)$ & $17(23.9)$ & 0.175 \\
\hline III/IV & $114(83.8)$ & $54(76.1)$ & \\
\hline \multicolumn{4}{|l|}{ B symptom } \\
\hline Yes & $87(64.0)$ & $42(59.2)$ & 0.497 \\
\hline No & $49(36.0)$ & $29(40.8)$ & \\
\hline \multicolumn{4}{|c|}{ Mediastinal mass } \\
\hline Yes & $46(33.8)$ & $32(45.1)$ & 0.113 \\
\hline No & $90(66.2)$ & $39(54.9)$ & \\
\hline \multicolumn{4}{|c|}{ LDH elevated } \\
\hline Yes & $86(63.2)$ & $30(42.3)$ & 0.004 \\
\hline No & $50(36.8)$ & $41(57.7)$ & \\
\hline \multicolumn{4}{|c|}{ Bone marrow invasion } \\
\hline Yes & $69(50.7)$ & $40(56.3)$ & 0.443 \\
\hline No & $67(49.3)$ & $31(43.7)$ & \\
\hline \multicolumn{4}{|c|}{ CNS involvement } \\
\hline Yes & $3(2.2)$ & $2(2.8)$ & $>0.99$ \\
\hline No & $133(97.8)$ & $69(97.2)$ & \\
\hline \multicolumn{4}{|l|}{ IPI } \\
\hline Low risk & $107(78.7)$ & $48(67.6)$ & 0.081 \\
\hline High risk & $29(21.3)$ & $23(32.4)$ & \\
\hline
\end{tabular}

Values are presented as number (\%). NHL-BFM-95, non-Hodgkin lymphoma Berlin-Frankfurt-Münster-95; HyperCVAD/ MA, hyperfractionated cyclophosphamide, vincristine, adriamycin, dexamethasone/methotrexate, and cytarabine; ECOG, Eastern Cooperative Oncology Group; T-LBL, T-lymphoblastic lymphoma; B-LBL, B lymphoblastic lymphoma; LDH, lactate dehydrogenase; CNS, central nervous system; IPI, international prognostic index.

primarily screened by univariate analysis and then evaluated by multivariate analysis. Due to limited cases, multivariate cox regression analysis was not performed to patients with B-LBL. Statistical analysis was performed with SPSS ver. 17.0 (SPSS Inc., Chicago, IL) and the figures were drawn with GraphPad Prism 7 (San Diego, CA).

\section{Ethical statement}

All procedures performed in studies involving human par- ticipants were in accordance with the ethical standards of the ethics committee and the institutional review board of two hospitals (Sun Yat-sen University Cancer Center and The First Affiliated Hospital of Hainan Medical College) (GYX2019-023) and with the 1964 Helsinki declaration and its later amendments or comparable ethical standards. Informed consent was obtained from all individual participants included in the study. 


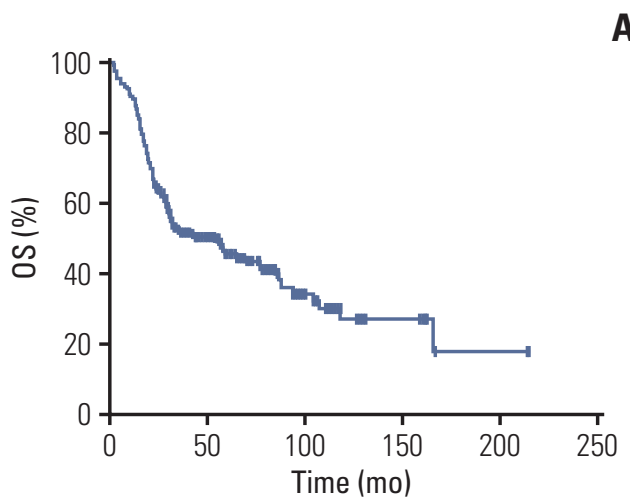

A

B

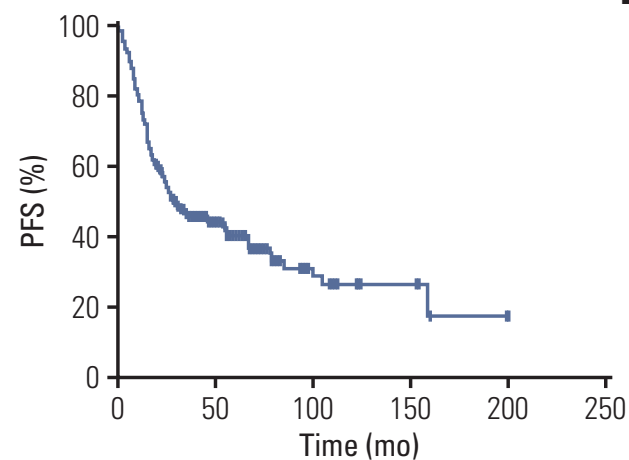

C
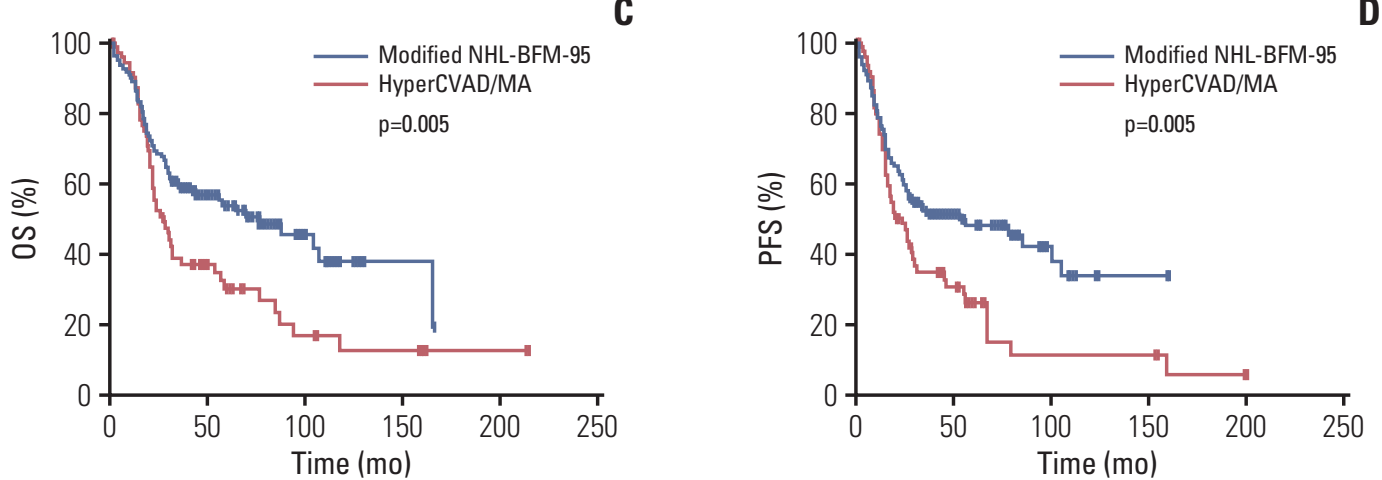

Fig. 1. (A, B) Kaplan-Meier curves for overall survival (OS) (A) and progression-free survival (PFS) (B) among 207 patients with lymphoblastic lymphoma (LBL). (C, D) Kaplan-Meier curves comparing OS (C) and PFS (D) between patients who received modified NHL-BFM-95 (non-Hodgkin lymphoma Berlin-Frankfurt-Münster-95) and HyperCVAD/MA (hyperfractionated cyclophosphamide, vincristine, adriamycin, dexamethasone/methotrexate, and cytarabine).

Table 3. Treatment outcomes of patients in different treatment groups

\begin{tabular}{|c|c|c|c|}
\hline Parameter & Modified NHL-BFM-95 & HyperCVAD/MA & p-value \\
\hline Total & 136 & 71 & \\
\hline CR after induction & $106(77.9)$ & $47(66.2)$ & 0.052 \\
\hline MRD negative after consolidation & $94(69.1)$ & $43(60.1)$ & 0.088 \\
\hline 5-Year OS & 53.9 & 30.2 & 0.005 \\
\hline 5-Year PFS & 47.9 & 25.9 & 0.005 \\
\hline Treatment-related mortality & 0 & 0 & \\
\hline \multicolumn{4}{|l|}{ Toxicity } \\
\hline Allergic reaction & $8(5.9)$ & $6(8.5)$ & \\
\hline Hypofibrinogenemia & $33(24.3)$ & $13(18.3)$ & \\
\hline Pancreatitis & $8(5.9)$ & $3(4.2)$ & \\
\hline Elevated liver enzymes & $47(34.6)$ & $33(46.5)$ & \\
\hline Elevated bilirubin & $27(19.9)$ & $16(22.5)$ & \\
\hline Osteonecrosis & $9(6.6)$ & $4(5.6)$ & \\
\hline Thrombosis & $19(14.0)$ & $12(16.9)$ & \\
\hline Stroke-like event & $4(2.9)$ & $2(2.8)$ & \\
\hline Neuropathy & $10(7.4)$ & $9(12.7)$ & \\
\hline
\end{tabular}

Values are presented as number (\%). NHL-BFM-95, non-Hodgkin lymphoma Berlin-Frankfurt-Münster-95; HyperCVAD / MA, hyperfractionated cyclophosphamide, vincristine, adriamycin, dexamethasone/methotrexate, and cytarabine; $\mathrm{CR}$, complete remission; MRD, minimal residual disease; OS, overall survival; PFS, progression-free survival. 
Table 4. Response to treatment in T-LBL and B-LBL patients according to treatment

\begin{tabular}{|c|c|c|c|c|c|c|c|c|}
\hline & \multicolumn{4}{|c|}{ T-LBL } & \multicolumn{4}{|c|}{ B-LBL } \\
\hline & CR $(\%)$ & p-value & $\begin{array}{c}\text { MRD } \\
\text { negative (\%) }\end{array}$ & p-value & CR $(\%)$ & p-value & $\begin{array}{c}\text { MRD } \\
\text { negative (\%) }\end{array}$ & p-value \\
\hline Modified NHL-BFM-95 $(\mathrm{n}=136)$ & 76.6 & 0.036 & 68.9 & 0.043 & 82.8 & 0.741 & 62.3 & 0.652 \\
\hline HyperCVAD/MA (n=71) & 64.0 & & 53.4 & & 71.4 & & 51.4 & \\
\hline Total $(\mathrm{n}=207)$ & 72.6 & & 63.4 & & 78.0 & & 58.7 & \\
\hline
\end{tabular}

T-LBL, T-lymphoblastic lymphoma; B-LBL, B lymphoblastic lymphoma; CR, complete remission; MRD, minimal residual disease; NHL-BFM-95, non-Hodgkin lymphoma Berlin-Frankfurt-Münster-95; HyperCVAD/MA, hyperfractionated cyclophosphamide, vincristine, adriamycin, dexamethasone/methotrexate, and cytarabine.

A

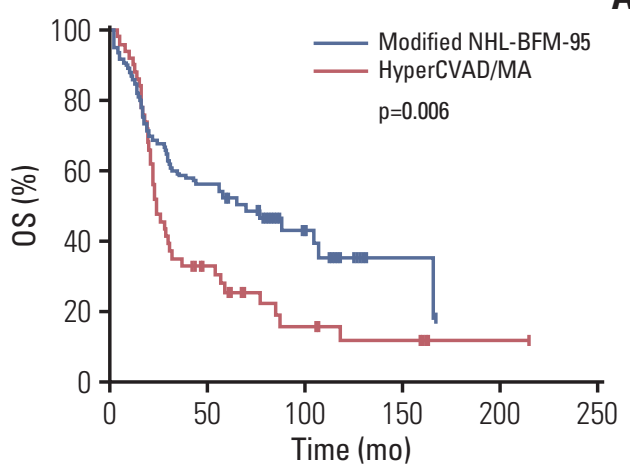

C

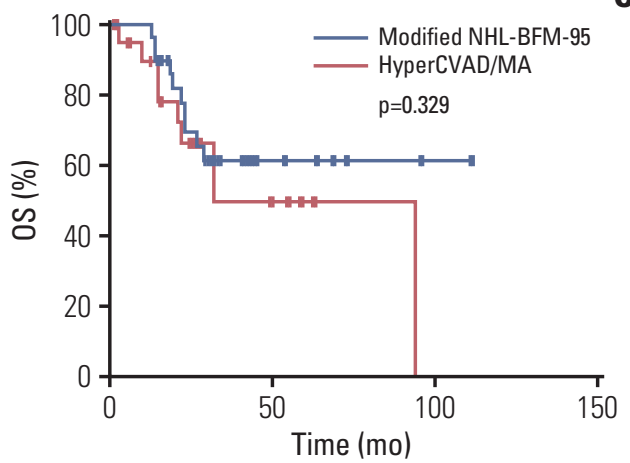

B

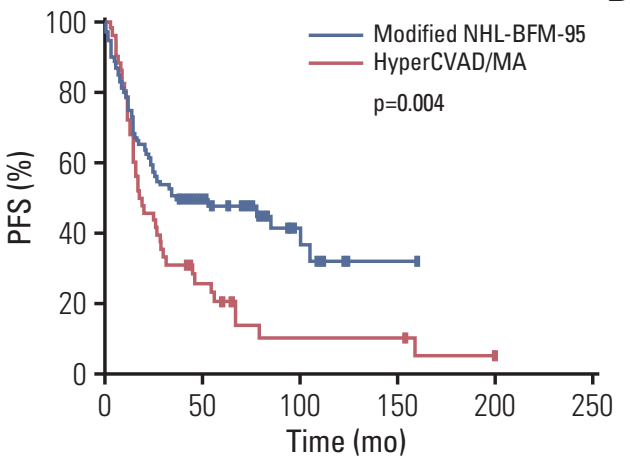

D

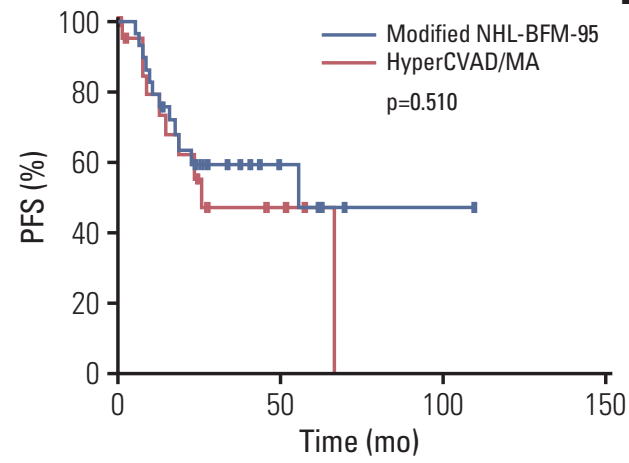

Fig. 2. Kaplan-Meier curves comparing overall survival (OS) and progression-free survival (PFS) between patients receiving modified NHL-BFM-95 (non-Hodgkin lymphoma Berlin-Frankfurt-Münster-95) regimen and those receiving HyperCVAD/MA (hyperfractionated cyclophosphamide, vincristine, adriamycin, dexamethasone/methotrexate, and cytarabine) according to different cell lineages. (A) OS of patients with T-LBL ( $\mathrm{p}=0.006$ ). (B) PFS of patients with T-LBL ( $\mathrm{p}=0.004)$. (C) OS of patients with B-LBL ( $\mathrm{p}=0.329$ ). (D) PFS of patients with B-LBL $(\mathrm{p}=0.510)$.

\section{Results}

\section{Clinical manifestation}

The clinical features of 207 LBL patients in two differently treated groups are recorded in Table 2. Sixty-five point seven percent of the patients were treated with modified NHLBFM-95 and 34.3\% were treated with HyperCVAD/MA. The median age was 28 years in patients treated with modified NHL-BFM-95 and 32 years in patients treated with HyperC$\mathrm{VAD} / \mathrm{MA}(\mathrm{p}=0.050)$. More patients were $<40$ years in the 
A
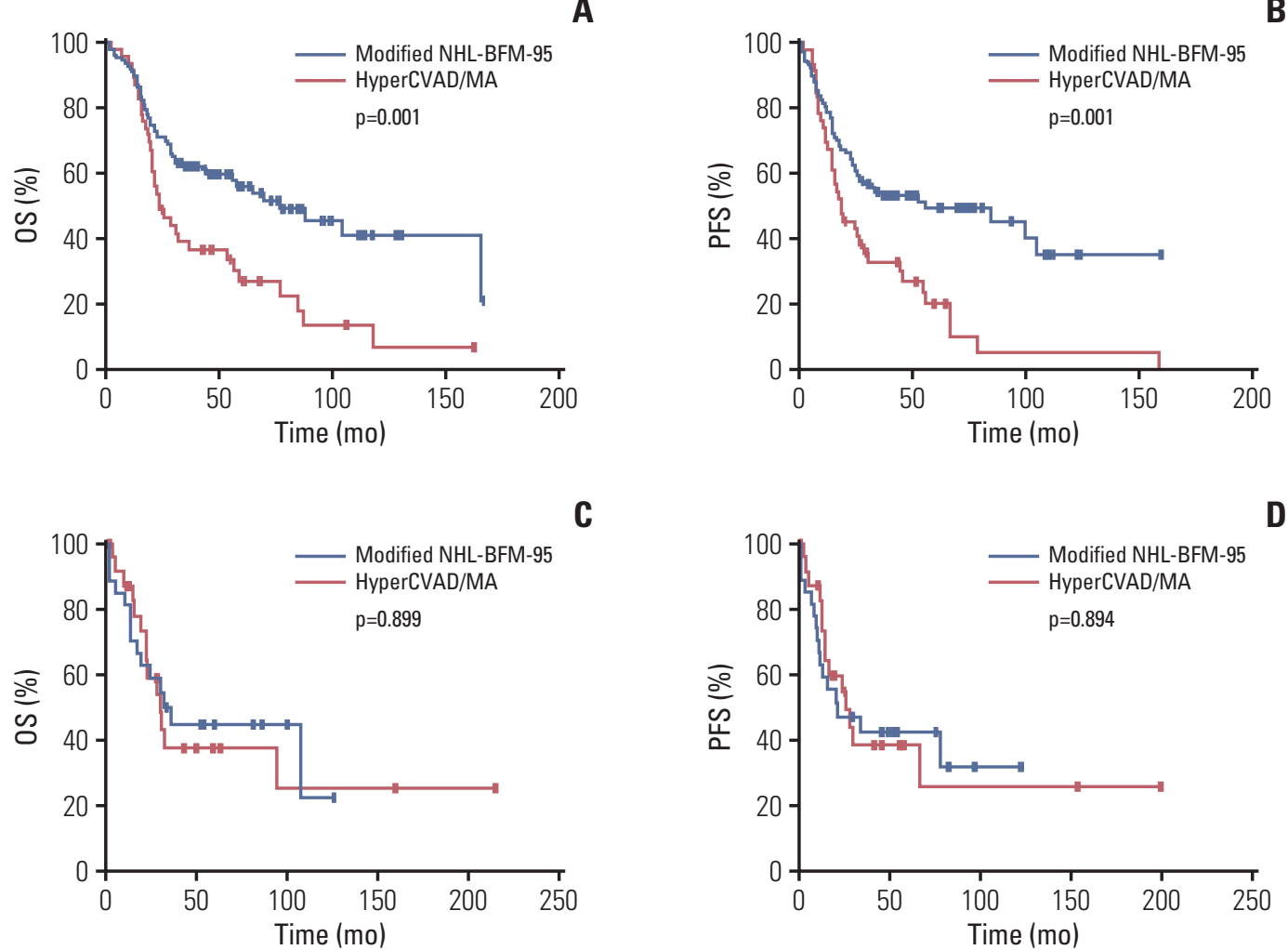

C

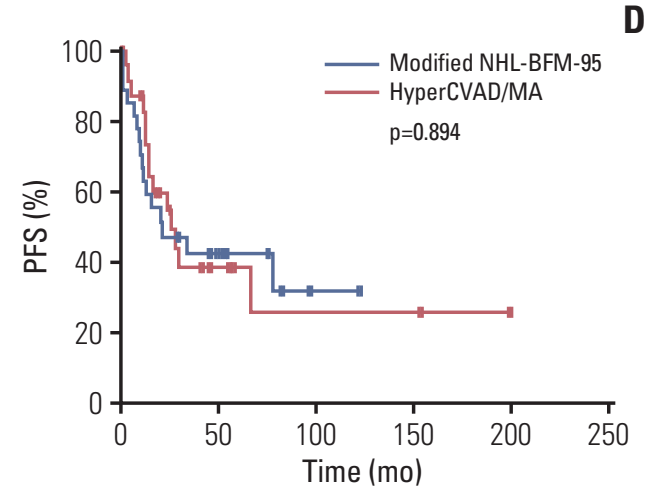

Fig. 3. Kaplan-Meier curves comparing overall survival (OS) and progression-free survival (PFS) between patients receiving modified NHL-BFM-95 (non-Hodgkin lymphoma Berlin-Frankfurt-Münster-95) regimen and those receiving HyperCVAD/MA (hyperfractionated cyclophosphamide, vincristine, adriamycin, dexamethasone/methotrexate, and cytarabine) according to age. (A) OS of adolescent and young adult population ( $\mathrm{p}=0.001)$. (B) PFS of adolescent and young adult population ( $\mathrm{p}=0.001)$. (C) OS of patients $>40$ years ( $\mathrm{p}=0.899)$. (D) PFS of patients $>40$ years $(\mathrm{p}=0.894)$.

modified NHL-BFM-95 group than the HyperCVAD/MA group $(80.1 \%$ vs. $64.8 \%, \mathrm{p}=0.016)$. In this study, serum lactate dehydrogenase (LDH) level was defined elevated when it was $>245 \mathrm{U} / \mathrm{L}$. LDH was more frequently found to be elevated in patients treated with modified NHL-BFM-95 than those treated with HyperCVAD/MA $(63.2 \%$ vs. $42.3 \%$, $\mathrm{p}=$ 0.004). Other clinical features including sex, immunophenotype, ECOG scores, stage, B symptom, mediastinal mass, BM invasion, CNS involvement, and IPI were similar between the two treatment groups.

\section{Treatment outcomes and survival}

The median duration of OS for LBL patients was 54.0 months (95\% confidence interval [CI], 31.5 to 76.5 months). The estimated 1-year, 3-year, 5-year and 7-year OS rates were $89.2 \% \pm 2.2 \%, 52.2 \% \pm 3.6 \%, 45.7 \% \pm 3.8 \%$, and $41.0 \% \pm 4.1 \%$, respectively. The median duration of PFS was 70.0 months $(95 \%$ CI, 42.2 to 97.8 months). The estimated 1-year, 3-year, 5-year and 7-year PFS rates were $76.0 \% \pm 3.0 \%, 46,3 \% \pm 3,6 \%, 40.3 \% \pm$
$3.8 \%$, and $33,2 \% \pm 4.3 \%$, respectively (Fig. $1 \mathrm{~A}$ and B).

The treatment outcomes of two treatment groups were demonstrated in Table 3. There were no significant differences between two groups concerning $\mathrm{CR}$ after induction and MRD negative after consolidation therapy. However, there was a tendency of higher $\mathrm{CR}$ and MRD negative rates in modified NHL-BFM-95 group than in the HyperCVAD group ( $77.9 \%$ vs. $66.2 \%, 69.1 \%$ vs. $60.1 \%$, respectively). 5-year OS and PFS were both significantly higher in patients treated with modified NHL-BFM-95 regimen (53.9\% vs. 30.2\%, $47.9 \%$ vs. $25.9 \%$, respectively, $\mathrm{p}=0.005$ ) (Fig. $1 \mathrm{C}$ and $\mathrm{D}$ ).

Table 4 demonstrated treatment response of two regimens in T-LBL and B-LBL patients. For patients with T-LBL, the $\mathrm{CR}$ and MRD negative rates between two treatment groups were significantly different $(p=0.036$ and $p=0.043$, respectively). Patients were more likely to achieve $\mathrm{CR}$ and MRD negative with modified NHL-BFM-95 regimen than HyperCVAD/MA (76.7\% vs. $64.0 \%, 68.9 \%$ vs. $53.4 \%$, respectively). In the meanwhile, for patients with B-LBL, the response between two treatment groups was not significantly different. 
Table 5. Prognostic factors associated with OS and PFS of T-LBL patients

\begin{tabular}{|c|c|c|c|c|c|c|}
\hline \multirow{3}{*}{$\begin{array}{l}\text { Prognostic } \\
\text { factor }\end{array}$} & \multicolumn{3}{|c|}{ Overall survival } & \multicolumn{3}{|c|}{ Progression-free survival } \\
\hline & \multirow{2}{*}{$\begin{array}{c}\begin{array}{c}\text { Univariate } \\
\text { analysis }\end{array} \\
\text { p-value }\end{array}$} & \multicolumn{2}{|c|}{$\begin{array}{c}\text { Multivariate } \\
\text { analysis }\end{array}$} & \multirow{2}{*}{$\begin{array}{c}\begin{array}{c}\text { Univariate } \\
\text { analysis }\end{array} \\
\text { P-value }\end{array}$} & \multicolumn{2}{|c|}{$\begin{array}{l}\text { Multivariate } \\
\text { analysis }\end{array}$} \\
\hline & & HR (95\% CI) & p-value & & HR $(95 \% \mathrm{CI})$ & p-value \\
\hline \multicolumn{7}{|l|}{ Treatment } \\
\hline $\begin{array}{l}\text { HyperCVAD/MA vs. } \\
\text { modified NHL-BFM-95 }\end{array}$ & 0.006 & $1.66(1.08-2.56)$ & 0.021 & 0.004 & $2.32(1.49-3.62)$ & $<0.001$ \\
\hline \multicolumn{7}{|l|}{ Age (yr) } \\
\hline$\geq 40$ vs. $<40$ & 0.429 & - & - & 0.401 & - & - \\
\hline \multicolumn{7}{|l|}{ Sex } \\
\hline Male vs. Female & 0.854 & - & - & 0.493 & - & - \\
\hline \multicolumn{7}{|l|}{ ECOG } \\
\hline$>2$ vs. $\leq 2$ & 0.038 & $2.37(1.38-4.07)$ & 0.002 & 0.040 & $2.16(1.25-3.73)$ & 0.006 \\
\hline \multicolumn{7}{|l|}{ WBC count at diagnose } \\
\hline Abnormal vs. Normal & 0.760 & - & - & 0.527 & - & - \\
\hline \multicolumn{7}{|l|}{ Stage } \\
\hline I/II vs. III/IV & 0.762 & - & - & 0.503 & - & - \\
\hline \multicolumn{7}{|l|}{ B symptom } \\
\hline Yes vs. No & 0.505 & - & - & 0.143 & - & - \\
\hline \multicolumn{7}{|l|}{ LDH elevated } \\
\hline Yes vs. No & 0.105 & - & - & 0.304 & - & - \\
\hline \multicolumn{7}{|l|}{ Bone marrow invasion } \\
\hline Yes vs. No & 0.002 & $2.71(1.68-4.38)$ & $<0.001$ & 0.028 & $2.06(1.27-3.32)$ & 0.003 \\
\hline \multicolumn{7}{|l|}{ IPI } \\
\hline Low risk vs. High risk & $<0.001$ & - & - & $<0.001$ & - & - \\
\hline
\end{tabular}

OS, overall survival; PFS, progression-free survival; T-LBL, T-lymphoblastic lymphoma; HR, hazard ratio; CI, confidence interval; NHL-BFM-95, non-Hodgkin lymphoma Berlin-Frankfurt-Münster-95; HyperCVAD/MA, hyper fractionated cyclophosphamide, vincristine, adriamycin, dexamethasone / methotrexate, and cytarabine; ECOG, Eastern Cooperative Oncology Group; WBC, white blood cell; LDH, lactate dehydrogenase; IPI, international prognostic index.

On the other hand, the impact of two regimens on survival was different between T-LBL and B-LBL. For patients with T-LBL, modified NHL-BFM-95 regimen produced a significant higher OS and PFS than those in HyperCVAD/MA group ( $p=0.006$ and $p=0.004$, respectively) (Fig. $2 A$ and $B$ ). In the meanwhile, for patients with B-LBL, there is no significant difference in OS and PFS between two regimens (Fig. 2C and D).

The outcomes of two regimens in different age group (patients $<40$ and $\geq 40$ years) was analyzed (Fig. 3). For patients $<40$ years, modified NHL-BFM- 95 regimen produced a significantly higher OS and PFS than those in HyperC$\mathrm{VAD} / \mathrm{MA}$ group ( $\mathrm{p}=0.001$ ) (Fig. $3 \mathrm{~A}$ and $\mathrm{B}$ ). In the meanwhile, for patients $\geq 40$ years, there is no significant difference of OS and PFS between two regimens (Fig. 3C and D). In summary, modified NHL-BFM-95 regimen produced better outcomes than HyperCVAD/MA in younger population.

\section{Univariate and multivariate analyses}

The prognostic factors for patients with T-LBL are summarized in Table 5. In univariate analysis, HyperCVAD/MA, high ECOG scores, BM invasion, and high IPI were adverse factors for OS and PFS. In the multivariable analysis, HyperCVAD/MA, high ECOG scores and BM involvement were independent risk factors for OS and PFS. The prognostic factors for patients with B-LBL are summarized in Table 6. In univariate analysis, elevated LDH level and high IPI were adverse prognostic factors for OS and PFS. Other factors, including age, sex, ECOG grades, white blood cell count, clinical stage, B symptoms, BM involvement and treatment, did not affect the outcome significantly.

\section{Toxicity}

All the patients who received modified NHL-BFM-95 or 
Table 6. Univariate analysis of prognostic factors for B lymphoblastic lymphoma patients

\begin{tabular}{|c|c|c|c|c|}
\hline \multirow{2}{*}{ Prognostic factor } & \multicolumn{2}{|c|}{ 5-Year OS } & \multicolumn{2}{|c|}{ 5-Year PFS } \\
\hline & Percent & p-value & Percent & p-value \\
\hline \multicolumn{5}{|l|}{ Treatment } \\
\hline Modified NHL-BFM-95 & 61.5 & 0.329 & 57.5 & 0.510 \\
\hline HyperCVAD/MA & 49.8 & & 47.4 & \\
\hline \multicolumn{5}{|l|}{ Age (yr) } \\
\hline$\geq 40$ & 55.4 & 0.997 & 43.3 & 0.919 \\
\hline$<40$ & 60.3 & & 57.7 & \\
\hline \multicolumn{5}{|l|}{ Sex } \\
\hline Male & 50.9 & 0.546 & 49.2 & 0.721 \\
\hline Female & 55.8 & & 48.6 & \\
\hline \multicolumn{5}{|l|}{ ECOG } \\
\hline$\leq 2$ & 58.6 & 0.429 & 49.1 & 0.533 \\
\hline$>2$ & - & & - & \\
\hline \multicolumn{5}{|l|}{ WBC count at diagnose } \\
\hline Normal & 59.7 & 0.596 & 53.9 & 0.687 \\
\hline Abnormal & 50.2 & & 46.4 & \\
\hline \multicolumn{5}{|l|}{ Stage at diagnosis } \\
\hline I/II & 61.4 & 0.651 & 54.5 & 0.585 \\
\hline III/IV & 56.7 & & 47.5 & \\
\hline \multicolumn{5}{|l|}{ B symptom } \\
\hline Yes & 58.3 & 0.725 & 54.0 & 0.599 \\
\hline No & 57.0 & & 42.6 & \\
\hline \multicolumn{5}{|l|}{ LDH elevated at diagnosis } \\
\hline Yes & 33.6 & $<0.001$ & 24.3 & $<0.001$ \\
\hline No & 94.7 & & 89.5 & \\
\hline \multicolumn{5}{|l|}{ Bone marrow invasion } \\
\hline Yes & 41.9 & 0.151 & - & 0.154 \\
\hline No & 61.8 & & 50.7 & \\
\hline \multicolumn{5}{|l|}{ IPI } \\
\hline Low risk & 66.1 & 0.003 & 64.9 & 0.016 \\
\hline High risk & 41.3 & & 37.5 & \\
\hline
\end{tabular}

OS, overall survival; PFS, progression-free survival; NHL-BFM-95, non-Hodgkin lymphoma Berlin-Frankfurt-Münster-95; ECOG, Eastern Cooperative Oncology Group; WBC, white blood cell; LDH, lactate dehydrogenase; IPI, international prognostic index.

HyperCVAD exhibited III/IV grade myelosuppression during remission induction. The myelosuppression included low WBC accounts of $0.05-0.7 \times 10^{9} / \mathrm{L}$, hemoglobin level of 23 $70 \mathrm{~g} / \mathrm{L}$ and platelet count of $1-20 \times 10^{9} / \mathrm{L}$. Fifty-eight patients developed serious infections and recovered after treatment stopped. No treatment-related mortality was observed in both treatment groups. Other side effects including allergic reaction, hypofibrinogenemia, pancreatitis, elevated liver enzymes and bilirubin, osteonecrosis, thrombosis, stroke-like event, and neuropathy were compared between two treatment groups in Table 3. No patients interrupted treatment for severe adverse events.

\section{Discussion}

As previously reported, encouraging results were obtained with ALL-type regimens in adult LBL patients. However, few studies directly compare the efficacies of two ALL-type regimens. The present study demonstrated a modified NHLBFM-95 regimen and compared with HyperCVAD in LBL patients. Our study found that the modified NHL-BFM-95 regimen produced superior outcomes than HyperCVAD in T-LBL patients, but not in B-LBL. Our study also analyzed the clinical features, outcome, and prognostic factors of 207 adult LBL patients diagnosed at two hospitals from 2000 to 
Table 7. Comparison of characteristics of modified NHL-BFM-95 and HyperCVAD with CALGB 10403

\begin{tabular}{lccc} 
Parameter & Modified NHL-BFM-95 & HyperCVAD/MA & CALGB 10403 \\
\hline No. & 136 & 71 & 295 \\
Median age (yr) & 28 & 32 & 24 \\
Age (\%) & & & 100 \\
$\quad<40$ & 80.1 & 64.8 & 0 \\
$\quad \geq 40$ & 19.9 & 35.2 & 100 \\
ECOG & & & 0 \\
$\quad \leq 2$ & 77.9 & 80.3 & No \\
$\quad>2$ & 22.1 & 19.7 & 31.3 \\
Organ dysfunction allowed & No & No & 24.1 \\
Ph-positive LBL (\%) & 0 & 0 & 89.0 \\
T-cell LBL (\%) & 78.7 & 70.4 & $44.0^{\mathrm{a})}$ \\
CR after induction (\%) & 77.9 & 66.2 & 73.0 \\
MRD negative after consolidation (\%) & 69.1 & 60.1 & EFS 59.0 \\
3-Year OS (\%) & 58.9 & 38.8 & 34.7 \\
3-Year PFS (\%) & 52.0 & & \\
\hline
\end{tabular}

NHL-BFM-95, non-Hodgkin lymphoma Berlin-Frankfurt-Münster-95; HyperCVAD /MA, hyperfractionated cyclophosphamide, vincristine, adriamycin, dexamethasone/methotrexate, and cytarabine; ECOG, Eastern Cooperative Oncology Group; LBL, lymphoblastic lymphoma; CR, complete remission; MRD, minimal residual disease; OS, overall survival; PFS, progression-free survival. a)Pretreatment samples for Ph chromosome were available in 131 of the 223 B-cell patients in the study of CALGB 10403.

2018.

The clinical characteristics of the LBL patients were analyzed in the present study. Most clinical characteristics were similar between the two treatment groups except for age and LDH level. Patients treated with modified NHL-BFM-95 regimen were more likely to be younger and with elevated LDH level. Age has not been identified as a significant prognostic factor in adult LBL in previous studies [7,9]. In a study on adult T-LBL [8], elevated LDH level was the only significant risk factor for poor survival. In summary, the predominance of elevated LDH level in the modified NHL-BFM-95 group was an unfavorable factor for patient outcomes.

In this study, the 5-year OS rate and the PFS rate of LBL patients were $45.7 \%$ and $40.3 \%$, respectively, and they were consistent with previous studies $[8,10]$. Table 3 showed that the 5-year OS and PFS rates of modified NHL-BFM-95 group were significantly higher than HyperCVAD/MA group, which was different with a previous report comparing an augmented BFM therapy with HyperCVAD in ALL patients [11]. This previous study reported that a 3-year OS of $74 \%$ in augmented BFM group versus $71 \%$ in HyperCVAD group in AYAs with ALL. The augmented BFM group was not associated with significant improvements in OS compared with HyperCAVD. The different study group and treatment regimen may contribute to different results.

Comparison of characteristics of modified NHL-BFM-95,
HyperCVAD with previously published AYA protocol CALGB 10403 [12] was demonstrated in Table 7. The present study enrolled patients with older age. All patients were under 40 years in the CALGB 10403 study and 19.9\% in the modified NHL-BFM-95 group were 40 or older. Patients in our study also had worse performance status. Thirty-one point three percent of the population in the CALGB 10403 study were Ph-positive; while, Ph-positive patients were excluded from our study. Besides, most of our enrollment were of T-cell lineage, but the situation was reversed in the CALGB 10403 study. The treatment outcomes of modified NHL-BFM-95 group, including CR rate, OS and PFS, were inferior compared with the CALGB 10403 study. The unfavorable results in our study could due to the generally worse characteristics of older age and poorer performance status of patients.

Seventy-two point six percent T-LBL patients and 78.0\% BLBL patients reached CR at the end of induction I; $63.4 \% \mathrm{~T}$ LBL patients and 58.7\% B-LBL patients reached MRD negative after consolidation regimen. The results were consistent with previous studies $[5,13]$. In our study, for T-LBL patients, they were more likely to achieve CR and MRD negative when treated with the modified NHL-BFM-95 regimen, suggesting that the modified NHL-BFM-95 regimen could be a favorable option for T-LBL. In the meanwhile, for patients with B-LBL, modified NHL-BFM-95 and hyper CVAD/MA 
produced similar outcomes. Different mechanisms of B-LBL may explain the results.

AYA patients (15-39 years) with cancer is considered as a unique population with different treatment response and clinical outcomes. In the present study, modified NHL-BFM95 regimen produced better outcomes than HyperCVAD / MA in patients $<40$ years. In the meanwhile, in the older age group, there was no significant difference between the outcomes of two regimens. The result was consistent with previous studies on ALL. Multiple studies have suggested that "pediatric" approaches (e.g., NHL-BFM regimen) produced better outcomes than "adult" approaches (e.g., hyper-CVAD regimen) in patients with ALL [14-19]. This present study added new evidence supporting modified NHL-BFM-95 in young adult LBL patients.

Risk factors have been discussed in many studies. Unlike the prognostic factors of ALL, strong and reliable prognostic factors of LBL have yet to be established. Most studies on adult LBL have identified age $>40$ years, male sex, increased LDH, BM or CNS infiltration, and high IPI as risk factors. However, these findings were inconsistent among studies $[9,20]$. In contrast to other reports $[8,11]$, our study found that sex did not influence T-LBL or B-LBL outcomes. In the present study, for patients with T-LBL, high ECOG scores, BM invasion, and HyperCVAD/MA affected OS and PFS. High ECOG scores contributed to a poor outcome, suggesting that the use of numerous supportive treatments could improve the prognosis of T-LBL. BM invasion negatively affected the outcome. Thus, more effective and novel therapy are needed, especially for patients with BM invasion. For patients with B-LBL, through univariate analysis, elevated LDH level and high IPI significantly influenced the OS and PFS rates. Prognostic factors were similar but not entirely identical between B-LBL and T-LBL, which could be explained by smaller series of B-LBL group. The treatment was an independent prognostic factor in T-LBL, not in B-LBL, suggesting that the modified NHL-BFM-95 regimen was a preferred treatment than HyperCVAD/MA for patients with T-LBL.

In the present study, we evaluated the efficacy of a modified regimen evolved from NHL-BFM-95 regimen. In induction therapy, we replaced daunorubicin by pirarubicin at same dose to avoid cardiac toxicity. Pegaspargase has lower immunogenicity and longer half-life and been a standard treatment in ALL therapy for children and adults [21-23]. Thus, in this study, L-asparaginase was substituted by pegaspargase as inductive drug for more convenient dosage regimen and potentially reduced immunogenicity. The common adverse reactions of pegaspargase, including hypersensitivity reactions, pancreatitis and thrombotic complications [22], were closely monitored. The adverse reactions related to pegaspargase observed in the present study were consistent with previously reported studies with adult ALL [24,25], indicating that the dosage of pegaspargase in our study was well-tolerated. CNS invasion at diagnosis is commonly observed in 3\%-15\% LBL patients [26,27], and even more frequent in relapsed patients, particularly without adequate CNS prophylaxis. In our study, five patients presented CNS invasion at diagnosis, and no patient experienced relapse with CNS involvement after treatment. The results suggested that intrathecal chemotherapy and additional four HD-MTX treatments in the modified NHL-BFM-95 regimen were adequate CNS protection.

This study reported one of the largest series of research on adult Chinese patients with LBL. The clinical characteristics and prognostic factors were analyzed. Our study showed that the modified NHL-BFM-95 regimen was a preferred treatment than HyperCVAD/MA for patients with T-LBL or young adult patients ( $<40$ years). Still, the survival rates of LBL were poor. Hence, novel treatments were demanded. Future randomized trials are warranted to evaluated modified NHL-BFM-95 regimen in larger population. We hope that our study can provide more insights into therapeutic strategies.

\section{Electronic Supplementary Material}

Supplementary materials are available at Cancer Research and Treatment website (https: // www.e-crt.org).

\section{Conflicts of Interest}

Conflict of interest relevant to this article was not reported.

\section{Acknowledgments}

We thank the patients and their families and all the investigators, including the physicians, nurses, and laboratory technicians in this study. This work was supported by PhD Start-up Fund of Natural Science Foundation of Guangdong Province under grant number: 2017A030310193; Young Teachers' Cultivation Project of Sun YatSen University under grant number 16ykpy30; Science and Technology Projects of Guangdong Province under grant number 2014A020212577; National Natural Science Foundation of China under grant number 81700148 .

\section{Author Details}

${ }^{1}$ Department of Hematologic Oncology, State Key Laboratory of Oncology in South China, Collaborative Innovation Center for Cancer Medicine, Sun Yat-sen University Cancer Center, Guangzhou, ${ }^{2}$ Department of Hematologic Oncology, The First Affiliated Hospital, Hainan Medical College, Haikou, China 


\section{References}

1. Bassan R, Maino E, Cortelazzo S. Lymphoblastic lymphoma: an updated review on biology, diagnosis, and treatment. Eur J Haematol. 2016;96:447-60.

2. Cortelazzo S, Ferreri A, Hoelzer D, Ponzoni M. Lymphoblastic lymphoma. Crit Rev Oncol Hematol. 2017;113:304-17.

3. Nathwani BN, Diamond LW, Winberg CD, Kim H, Bearman RM, Glick JH, et al. Lymphoblastic lymphoma: a clinicopathologic study of 95 patients. Cancer. 1981;48:2347-57.

4. Jost LM, Jacky E, Dommann-Scherrer C, Honegger HP, Maurer R, Sauter C, et al. Short-term weekly chemotherapy followed by high-dose therapy with autologous bone marrow transplantation for lymphoblastic and Burkitt's lymphomas in adult patients. Ann Oncol. 1995;6:445-51.

5. Le Gouill S, Lepretre S, Briere J, Morel P, Bouabdallah R, Raffoux $\mathrm{E}$, et al. Adult lymphoblastic lymphoma: a retrospective analysis of 92 patients under 61 years included in the LNH87/ 93 trials. Leukemia. 2003;17:2220-4.

6. Thomas DA, O'Brien S, Cortes J, Giles FJ, Faderl S, Verstovsek $S$, et al. Outcome with the hyper-CVAD regimens in lymphoblastic lymphoma. Blood. 2004;104:1624-30.

7. Lepretre S, Touzart A, Vermeulin T, Picquenot JM, TanguySchmidt A, Salles G, et al. Pediatric-like acute lymphoblastic leukemia therapy in adults with lymphoblastic lymphoma: the GRAALL-LYSA LL03 study. J Clin Oncol. 2016;34:572-80.

8. Hoelzer D, Gokbuget N, Digel W, Faak T, Kneba M, Reutzel $\mathrm{R}$, et al. Outcome of adult patients with T-lymphoblastic lymphoma treated according to protocols for acute lymphoblastic leukemia. Blood. 2002;99:4379-85.

9. Cortelazzo S, Intermesoli T, Oldani E, Ciceri F, Rossi G, Pogliani EM, et al. Results of a lymphoblastic leukemia-like chemotherapy program with risk-adapted mediastinal irradiation and stem cell transplantation for adult patients with lymphoblastic lymphoma. Ann Hematol. 2012;91:73-82.

10. Neth O, Seidemann K, Jansen P, Mann G, Tiemann M, Ludwig WD, et al. Precursor B-cell lymphoblastic lymphoma in childhood and adolescence: clinical features, treatment, and results in trials NHL-BFM 86 and 90. Med Pediatr Oncol. 2000;35: 20-7.

11. Rytting ME, Thomas DA, O'Brien SM, Ravandi-Kashani F, Jabbour EJ, Franklin AR, et al. Augmented Berlin-Frankfurt-Munster therapy in adolescents and young adults (AYAs) with acute lymphoblastic leukemia (ALL). Cancer. 2014;120: 3660-8.

12. Stock W, Luger SM, Advani AS, Yin J, Harvey RC, Mullighan $\mathrm{CG}$, et al. A pediatric regimen for older adolescents and young adults with acute lymphoblastic leukemia: results of CALGB 10403. Blood. 2019;133:1548-59.

13. Wang K, Chen X, Wuxiao Z, Wang Z, Sun X, Zeng Z, et al. Long-term outcomes of modified Berlin-Frankfurt-Munster90 regimen in adults with T-lymphoblastic lymphoma: a single-center experience. Leuk Lymphoma. 2014;55:1800-5.

14. Stock W, La M, Sanford B, Bloomfield CD, Vardiman JW, Gaynon P, et al. What determines the outcomes for adolescents and young adults with acute lymphoblastic leukemia treated on cooperative group protocols? A comparison of Children's Cancer Group and Cancer and Leukemia Group B studies. Blood. 2008;112:1646-54.

15. Hallbook H, Gustafsson G, Smedmyr B, Soderhall S, Heyman M; Swedish Adult Acute Lymphocytic Leukemia Group, et al. Treatment outcome in young adults and children $>10$ years of age with acute lymphoblastic leukemia in Sweden: a comparison between a pediatric protocol and an adult protocol. Cancer. 2006;107:1551-61.

16. Hayakawa F, Sakura T, Yujiri T, Kondo E, Fujimaki K, Sasaki $\mathrm{O}$, et al. Markedly improved outcomes and acceptable toxicity in adolescents and young adults with acute lymphoblastic leukemia following treatment with a pediatric protocol: a phase II study by the Japan Adult Leukemia Study Group. Blood Cancer J. 2014;4:e252.

17. Ruiz-Delgado GJ, Macias-Gallardo J, Lutz-Presno JA, MontesMontiel M, Ruiz-Arguelles GJ. Outcome of adults with acute lymphoblastic leukemia treated with a pediatric-inspired therapy: a single institution experience. Leuk Lymphoma. 2011; 52:314-6.

18. Alacacioglu I, Medeni SS, Ozsan GH, Payzin B, Sevindik OG, Acar $\mathrm{C}$, et al. Is the BFM regimen feasible for the treatment of adult acute lymphoblastic leukemia? A retrospective analysis of the outcomes of BFM and Hyper-CVAD chemotherapy in two centers. Chemotherapy. 2014;60:219-23.

19. El-Cheikh J, El Dika I, Massoud R, Charafeddine M, Mahfouz R, Kharfan-Dabaja MA, et al. Hyper-CVAD compared with BFM-like chemotherapy for the treatment of adult acute lymphoblastic leukemia: a retrospective single-center analysis. Clin Lymphoma Myeloma Leuk. 2017;17:179-85.

20. Kobayashi R, Takimoto T, Nakazawa A, Fujita N, Akazai A, Yamato K, et al. Inferior outcomes of stage III T lymphoblastic lymphoma relative to stage IV lymphoma and T-acute lymphoblastic leukemia: long-term comparison of outcomes in the JACLS NHL T-98 and ALL T-97 protocols. Int J Hematol. 2014;99:743-9.

21. Avramis VI, Sencer S, Periclou AP, Sather H, Bostrom BC, Cohen LJ, et al. A randomized comparison of native Escherichia coli asparaginase and polyethylene glycol conjugated asparaginase for treatment of children with newly diagnosed standard-risk acute lymphoblastic leukemia: a Children's Cancer Group study. Blood. 2002;99:1986-94.

22. Place AE, Stevenson KE, Vrooman LM, Harris MH, Hunt SK, O'Brien JE, et al. Intravenous pegylated asparaginase versus intramuscular native Escherichia coli L-asparaginase in newly diagnosed childhood acute lymphoblastic leukaemia (DFCI 05-001): a randomised, open-label phase 3 trial. Lancet Oncol. 2015;16:1677-90.

23. Douer D, Aldoss I, Lunning MA, Burke PW, Ramezani L, Mark L, et al. Pharmacokinetics-based integration of multiple doses of intravenous pegaspargase in a pediatric regimen for adults with newly diagnosed acute lymphoblastic leukemia. J Clin Oncol. 2014;32:905-11.

24. Liang J, Shi P, Guo X, Li J, He L, Wang Y, et al. A retrospective 
comparison of Escherichia coli and polyethylene glycol-conjugated asparaginase for the treatment of adolescents and adults with newly diagnosed acute lymphoblastic leukemia. Oncol Lett. 2018;15:75-82.

25. Stock W, Douer D, DeAngelo DJ, Arellano M, Advani A, Damon L, et al. Prevention and management of asparaginase/ pegasparaginase-associated toxicities in adults and older adolescents: recommendations of an expert panel. Leuk Lymphoma. 2011;52:2237-53.
26. Song KW, Barnett MJ, Gascoyne RD, Chhanabhai M, Forrest DL, Hogge DE, et al. Primary therapy for adults with T-cell lymphoblastic lymphoma with hematopoietic stem-cell transplantation results in favorable outcomes. Ann Oncol. 2007; 18:535-40.

27. Bersvendsen H, Kolstad A, Blystad AK, Aurlien E, Fossa A, Kvaloy SO, et al. Multimodal treatment with ALL-like chemotherapy, Auto-SCT and radiotherapy for lymphoblastic lymphoma. Acta Oncol. 2014;53:680-7. 